\title{
The national MD-PhD program outcomes study: Relationships between medical specialty, training duration, research effort, and career paths
}

\author{
Lawrence F. Brass ${ }^{1}$ and Myles H. Akabas ${ }^{2}$ \\ 'Departments of Medicine and Pharmacology, University of Pennsylvania Perelman School of Medicine, Philadelphia, \\ Pennsylvania, USA. ${ }^{2}$ Department of Physiology \& Biophysics, Neuroscience, and Medicine, Albert Einstein College of \\ Medicine, Bronx, New York, USA.
}

MD-PhD programs were established in the 1950 s as a new curriculum for training physicianscientists. Since then, the number of programs has grown considerably; however, concerns about the health of the US physician-scientist workforce have grown, as well. The largest attempt to date to assess whether MD-PhD programs are fulfilling their mission was the national MD-PhD program outcomes study, which was released as an American Association of Medical Colleges report in 2018. That study gathered information on 10,591 graduates of $80 \mathrm{MD}-\mathrm{PhD}$ programs over $\mathbf{5 0}$ years and concluded that most graduates have followed careers consistent with their training. Here, we provide additional analysis, drawing on survey data provided by $64.1 \%$ of alumni $(75.9 \%$ of alumni with valid email addresses), plus program-supplied current workplace data for survey nonresponders to examine the relationships between medical specialty choices, training duration, research effort, and success in obtaining research funding. The results show that residency choices affect critical aspects of the physician-scientist career path, including where graduates work, how long it takes them to obtain an independent appointment in academia, and the amount of their professional time that is devoted to research. Entrants into MD-PhD programs are older, on average, now than when the programs were first established and are taking longer to graduate and complete postgraduate training. Although we found a positive relationship between professional effort devoted to research and the likelihood of having research funding, we found little evidence that the increase in training duration produces an increase in subsequent research effort. These data should provide both guidance for anyone considering this career path and insights for those who train and hire the next generation of physician-scientists.

Conflict of interest: The authors have declared that no conflict of interest exists.

Copyright: () 2019, American Society for Clinical Investigation.

Reference information: /CI Insight. 2019;4(19):e133009.

https://doi.org/10.1172/jci. insight.133009.

\section{Introduction}

Over the past century, biomedical research has expanded our understanding of biological processes from the molecular to the systems level and produced new therapeutic approaches. As experts in both clinical medicine and rigorous scientific research, physician-scientists are well positioned to play an important role in bringing the benefits of research to patients and clinical perspectives to the laboratory. Despite their successes, however, concerns have arisen about the viability of the US physician-scientist workforce and the practicality of a career that attempts to encompass both research and clinical care (1-5).

Historically, there have been multiple paths to a career as a physician-scientist. In the 1950s, a handful of medical schools and the NIH explored ways to combine clinical and research training, leading to the establishment, in 1964, of competitive National Institute of General Medical Sciences (NIGMS) Medical Scientist Training Program (MSTP) institutional T32 awards for programs that combine graduate school with medical school (6). Initially, there were very few $\mathrm{MD}-\mathrm{PhD}$ programs, but fueled by NIH training dollars and the enthusiastic participation of research-oriented medical schools, the number of programs has increased greatly, as has the total number of students enrolled (7).

As programs have expanded and training costs have grown, a number of studies have attempted to assess program effectiveness (7-13). Some studies have focused on specific metrics, such as NIH 
research grants, while others have defined success more broadly. However, all have been limited by the number of alumni analyzed and the time over which follow-up data were available. To address these limitations, the MD-PhD section of the American Association of Medical Colleges (AAMC) Group on Graduate Research Education and Training (GREAT), in partnership with AAMC research staff members, organized a survey of the 10,591 known graduates of $80 \mathrm{MD}-\mathrm{PhD}$ programs throughout the United States and combined the survey results with alumni information in AAMC databases. The results were released as an AAMC report in April 2018 (14). Key findings showed that most program graduates were following careers consistent with the goals of their training. However, the data also indicated that training time has steadily increased. Here, we provide additional analysis, drawing on survey data provided by $64 \%$ of program alumni plus current workplace data on survey nonresponders provided by participating programs to examine the relationships between graduate medical education (GME) choices, total training duration (during and after residency), research effort, and success in obtaining research funding. In an accompanying manuscript (15), we address the impact of sex, race, and ethnicity on career outcomes.

\section{Methods}

In 2013-2014, $80 \mathrm{MD}-\mathrm{PhD}$ programs, including 44 of the 45 programs that were receiving MSTP T32 funding at the time, provided names along with matriculation and graduation dates of all of their program graduates to the AAMC Data Operations and Services. In total, 10,591 graduates of the 80 participating $\mathrm{MD}-\mathrm{PhD}$ programs were identified, and their unique AAMC identification numbers were determined. This allowed cross-referencing of survey responses and information on the individuals in various AAMC databases. The programs provided valid email addresses for 8,944 (84.4\%) of the graduates. The survey was approved by AAMC IRB. Prior to launching the survey, each graduate received an email from the director of the program from which they graduated, informing them that the program was participating in a national $\mathrm{MD}-\mathrm{PhD}$ program outcomes study and that they would receive an email from AAMC with an active URL link to participate in the study. At the start of the survey, participants were asked for consent to participate. At approximately monthly intervals for 3 months after the first email, nonresponders received a follow-up email from the program director and then an email from AAMC, requesting their participation. The survey closed in June 2015. By that time, 6,786 alumni had completed the survey $(64.1 \%$ of all alumni and $75.9 \%$ of alumni with valid email addresses). Survey response data were integrated with information from AAMC databases on all graduates, and the deidentified person-level responses of survey responders were provided to the authors for further analysis. A more detailed description of the survey process and a copy of the survey can be found in the AAMC report (14).

Survey nonresponders. To obtain information about the current position of the 3,805 graduates for whom the programs either lacked valid email addresses (1,647 graduates) or who did not respond to the survey (2,158 graduates), we asked each program director to categorize the current position of these individuals and return the deidentified aggregated information. This was possible because all of the MD-PhD programs are expected to track the careers of their graduates, obtaining the information either from the graduate directly or from publicly available sources. Herein, we refer to these 3,805 graduates as the nonresponders. We also asked the programs to identify the number of trainees who were retired or deceased and to categorize them by their final type of workplace prior to retirement or death. Thirty-four programs, including 22 MSTP-funded programs, provided information on 1,888 individuals $(49.6 \%$ of the nonresponders). This information was excluded from the AAMC report, which only included an analysis of survey responders, but has been used here to determine whether the information obtained from survey responders is representative of those who did not respond.

Data analysis. Deidentified information released to us by the AAMC was provided in the form of Microsoft Excel spreadsheets that included birth year, years of matriculation and graduation, sex, race, ethnicity, employment status, residency field, initial and current workplace type, academic rank, distribution of professional effort, and research awards. Age at matriculation was calculated as the year of matriculation minus the birth year. When indicated, comparisons were made using a 1-way ANOVA using either Dunnett's multiple comparison test to compare multiple groups to a single group or Tukey's multiple comparisons test to compare all data points to each other (GraphPad Prism 7). 
A Survey responders

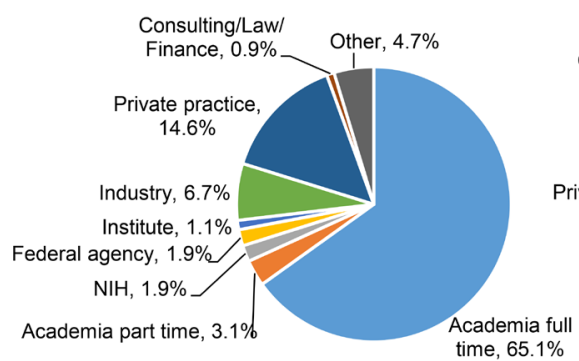

B Non-responders

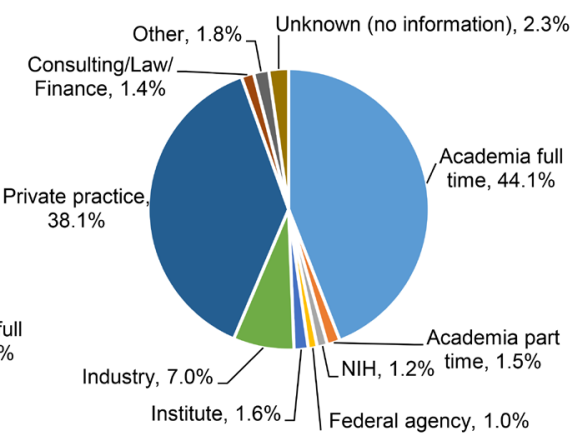

C Combined

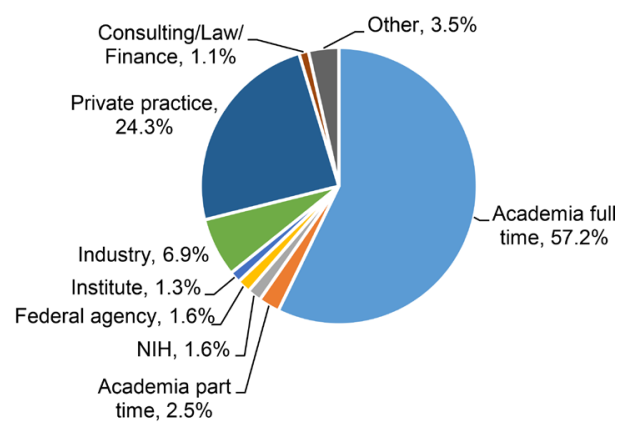

Figure 1. Current primary workplace of MD-PhD program graduates who have completed postgraduate training. Current workplace of (A) 4,636 survey responders and (B) 1,888 survey nonresponders based on data provided by 34 program directors out of 3,805 nonresponders, and (C) the weighted average of the percentage in each workplace category from the data in $\mathbf{A}$ and $\mathbf{B}$.

\section{Results}

Workplace choices. Program alumni were asked to categorize their first independent position after postgraduate training and their current position. A total of $65 \%$ of survey responders reported their current position as being in academia full time (Figure 1A); $80 \%$ were in academia, the NIH, a federal agency other than the $\mathrm{NIH}$, research institutes, or industry. Only $15 \%$ reported being in private practice.

To determine whether the survey responders are representative of the nonresponders, we asked program directors to use the same categories to identify the current positions of their graduates who had not completed the survey. The results were provided to us in aggregate from each program without identifying any of the graduates. Of the 80 programs participating in the survey, 34 provided information on 1,888 graduates, which is $49.6 \%$ of the 3,805 nonresponders. Figure $1 \mathrm{~B}$ summarizes the available data on nonresponders. The percentage of trainees in academia was substantially lower among nonresponders than responders ( $44 \% \mathrm{vs}$. $65 \%$ ), and the percentage of nonresponders in private practice was substantially higher (38\% vs. $15 \%)$. If we assume that the data provided to us by program directors on the nonresponders is representative of the entire cohort of nonresponders, we can generate a weighted average for the percentage in each category. This extrapolation indicates that $71 \%$ of graduates out of training hold positions in academia, the NIH or other federal agency, or research institutes or industry, and that $24 \%$ are in private practice (Figure 1C).

How reliable is this extrapolation? To answer this question for individuals in academia, we used data from a search of the AAMC Faculty Roster (FR) for the 8,276 program alumni who were not listed in the AAMC GME Track database as being actively in training in 2014 (14). FR data are provided by medical schools affiliated with the AAMC and are, therefore, independent of the survey used for this study. In the MD-PhD program outcomes study, the FR search found $66 \%$ of survey responders, $45 \%$ of nonresponders, and $58 \%$ of all alumni (14). These numbers are strikingly similar to the corresponding numbers for those in academia in Figure 1, A-C (65\%, 44\%, and 57\%, respectively). This suggests that the extrapolated results shown in Figure $1 \mathrm{C}$ are reasonably accurate, at least with respect to having a work place in academia.

Workplace choice and research effort. In addition to doing research, medical school faculty are called upon to see patients, teach, and serve as administrators. In the survey, we asked alumni to indicate how they divide their time among these and other activities. The definition of teaching provided in the survey included classroom lectures, small group preceptorships, and teaching in the clinical setting. Time spent teaching students and postdocs in their laboratory was defined as research time. The only constraint we placed on this determination was that the sum of efforts in all areas should be equal to $100 \%$.

Figure 2A summarizes the survey responses from 3,030 alumni working in academia full-time, grouping the results by research effort and displaying the data by deciles (Supplemental Figure 1; supplemental material available online with this article; https://doi.org/10.1172/jci.insight.133009DS1). Two conclusions stand out. First, research effort in academia rarely falls into the 80:20 distribution with clinical effort that is sometimes touted as the optimal mix for a physician-scientist. Instead, the research effort plot shows that the percent of time spent on research encompasses nearly all possible values between $0 \%$ and $100 \%$. Second, as research effort decreases, effort devoted to other activities increases, but the increase is, on average, predominantly in clinical effort. 
A

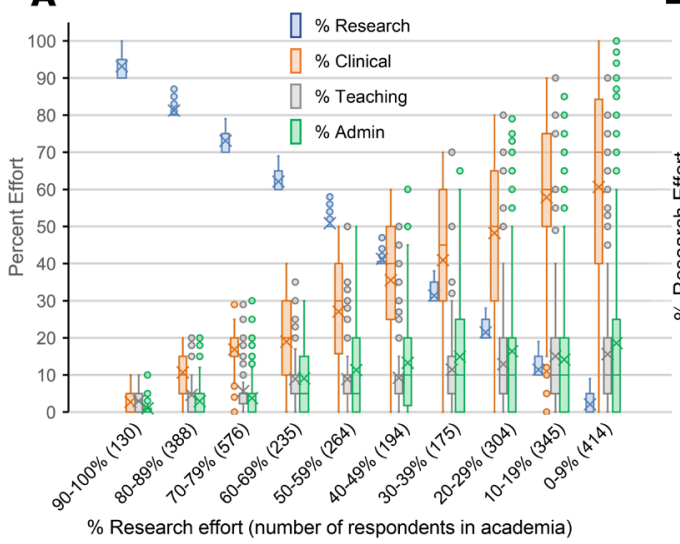

B

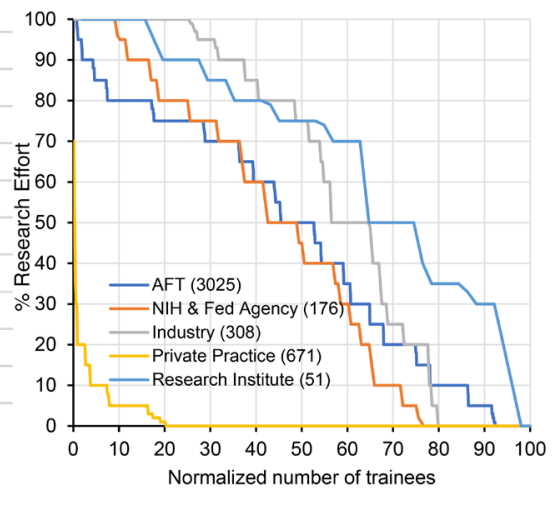

C

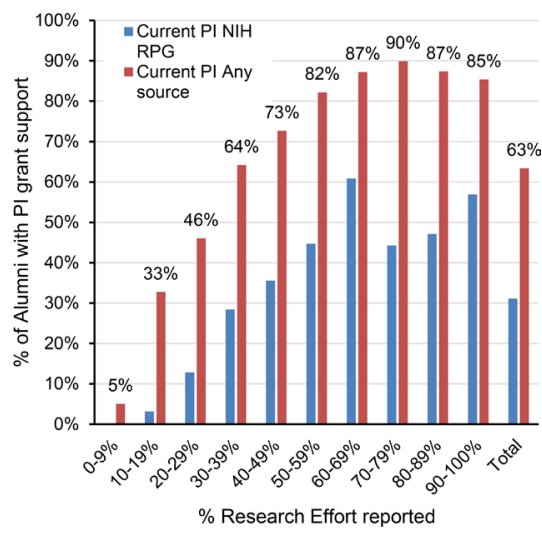

Figure 2. Distribution of reported research and clinical effort. (A) Percent effort reported for research, clinical, teaching, and administrative effort for 3,017 survey responders with a current position in academia full-time broken down by research effort deciles. Boxes indicate the second and third quartiles. Whiskers are drawn using Tukey's criteria of $1.5 \times$ the interquartile range. Outliers beyond the whiskers are shown. $\mathrm{X}$ indicates the average. Horizontal bar in the box indicates the median. In Supplemental Figure 1, the data are displayed in bar graph format showing the mean \pm SEM. (B) Research effort for individual alumni in each of the current workplaces shown. Alumni are rank ordered from greatest reported percent research effort to least. AFT, academia full-time. The $X$ axis has been normalized by the number of alumni to allow comparisons between workplaces that vary considerably in the number of alumni in each ( $n$ values are in parentheses). By 1-way ANOVA using Dunnett's multiple comparisons test, the average research effort of those in industry and research institutes is significantly greater than those in AFT $(P<0.001)$, and the average of those in private practice is significantly less than those in AFT $(P<0.001)$. The average research effort of those in AFT and at NIH and other federal agencies is similar. (C) Percent of alumni in academia full-time reporting that they were a principal investigator (PI) with current grant support from either any source (red bars) or a NIH research project grant (RPG) (blue bars) as a function of reported percent research effort. Percentage above each pair of bars indicates the percent of those with PI grant support who are PI on NIH RPG as part of their grant portfolio.

Figure $2 \mathrm{~B}$ compares the rank-ordered research effort by individual trainees in each current workplace, normalizing the data because of the difference in the number of trainees in each type of workplace. The results reflect the wide variation in individual research effort among those in academia, the $\mathrm{NIH}$ and other federal agencies, industry, private practice, and research institutes. Those working at research institutes or in industry reported the greatest overall research effort. Those in academia and at federal agencies reported less research effort. Those in private practice devoted by far the least effort to research, with most reporting none, challenging the assumption that they are necessarily using their research training to participate in clinical trials.

Research effort and research awards. Overall, $63 \%$ of survey responders working in academia full-time reported that they were currently principal investigators on research awards from any source. This number was much higher (87\%) among those with $\geq 50 \%$ research effort $(n=1,593)$ and lower $(37 \%)$ among those with $<50 \%$ research effort $(n=1,433)$. The proportion of grant holders whose funding portfolio included an NIH research award rose from $0 \%$ for those with $<10 \%$ research effort to as high as $70 \%$ for those with $\geq 50 \%$ research effort (Figure 2C). In general, the greater the research effort, the greater the likelihood that a program graduate working in academia had research funding and the greater the likelihood that that funding included NIH support. Of note, however, this relationship leveled off at approximately $50 \%$ research effort. Moreover, because the survey asked about awards, but not award size, these results do not exclude the possibility that the amount of funding continues to increase as research effort increases above $50 \%$.

Residency choices. Of the 6,786 alumni who responded to the survey, 4,655 had completed postgraduate training, including $356(7.6 \%)$ who did not do postgraduate clinical training. The distribution of residency choices made by the 4,299 graduates who had completed a residency at the time of the survey is shown in Figure 3. Nearly $60 \%$ trained in internal medicine, pathology, or pediatrics. An additional $25 \%$ trained in neurology, surgery, psychiatry, and radiology. A comparison with data on medical school graduates in general shows that $\mathrm{MD}-\mathrm{PhD}$ program alumni were more likely to choose internal medicine (27\% vs. $22 \%)$ and pathology (14\% vs. $1 \%)$ and less likely to choose surgery $(7.4 \%$ vs. $12 \%)(16)$. Previous studies of $\mathrm{MD}-\mathrm{PhD}$ program alumni have found that medical specialty choices have changed over time $(7,17)$. The survey results were consistent with this finding. For example, the percentage of alumni training in internal 


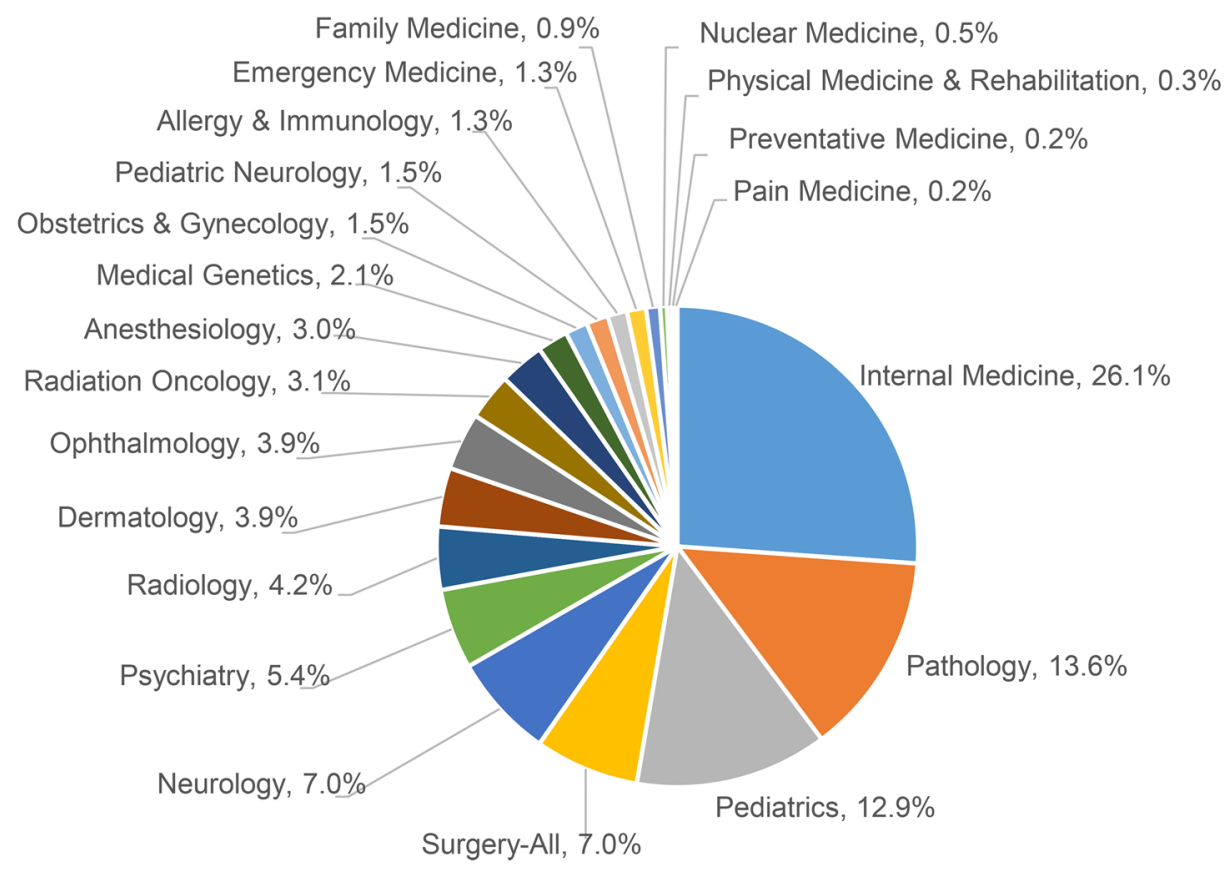

Figure 3. Distribution of graduate medical specialty training choices of the 4,297 survey responders who have completed postgraduate training. The 362 individuals who indicated that they did not do postgraduate clinical training have been excluded from the figure. There were 211 individuals who reported completing GME training in more than 1 specialty. The percentages were calculated based on the total number of GME specialties selected, 4,508 $(4,297+211)$

medicine was $40 \%$ in the cohort that graduated prior to 1975 , fell to $21 \%$ in the cohort that graduated from 2005-2014, and then rose to $27 \%$ among those who were in training at the time of the survey (14).

Residency field and workplace choice. A total of $65 \%$ of survey responders who had completed postgraduate training indicated that their workplace at the time of the survey was in academia (Figure 1A). However, this number ranged from $31 \%$ in family medicine to $85 \%$ in pediatric neurology, at least among survey responders (Figure 4). Supplemental Figure 2A and Supplemental Figure 3A show the percentage of alum$\mathrm{ni}$ in academia who are in internal medicine or pediatrics broken down by subspecialty. In both cases, there was considerable variation, ranging from approximately $50 \%-100 \%$. Those who did not report having completed subspecialty training generally had the lowest percentage with a current position in academia.

Residency field and training duration. Total training duration for physician-scientists includes postgraduate training time (residencies, fellowships, and postdocs) as well as time to degree. The survey data show that both have increased, confirming results on time to degree published previously (7). In the national MD-PhD program outcomes study, the cohort that graduated between 1975 and 1984 required 6.69 years on average to complete both degrees. The cohort that graduated between 2005 and 2014 required 8.25 years, a nearly $25 \%$ increase (Figure $5 \mathrm{~A}$ ). At the same time, the average age at matriculation into an MD-PhD program increased from 21.9 years before 1975 to 23.1 for the cohort graduating 2005-2014.

To obtain information on postgraduate training duration, the survey asked responders to indicate how many years passed between their MD-PhD graduation and their first appointment to a full-time position after residency/fellowship/postdoc as a physician and/or scientist. The data show that the median time to employment either in academia, NIH or other federal agency, the pharmaceutical or biotech industry, or a nongovernmental research institute rose from approximately 3 years for the cohort of alumni graduating before 1975 to 6 years for those graduating between 1995-2004 (Figure 5B). We chose not to include more recent graduates because many of them were still in training. Since some responders based their answer on their first appointment as an assistant professor and others included their first appointment as an instructor, the numbers in Figure 5B are, if anything, an underestimate.

In Figure 5C, we subdivided the survey data for the 1995-2004 cohort by medical specialty. The results show considerable variation in the time to first position in academia, with anesthesiology taking the least time 


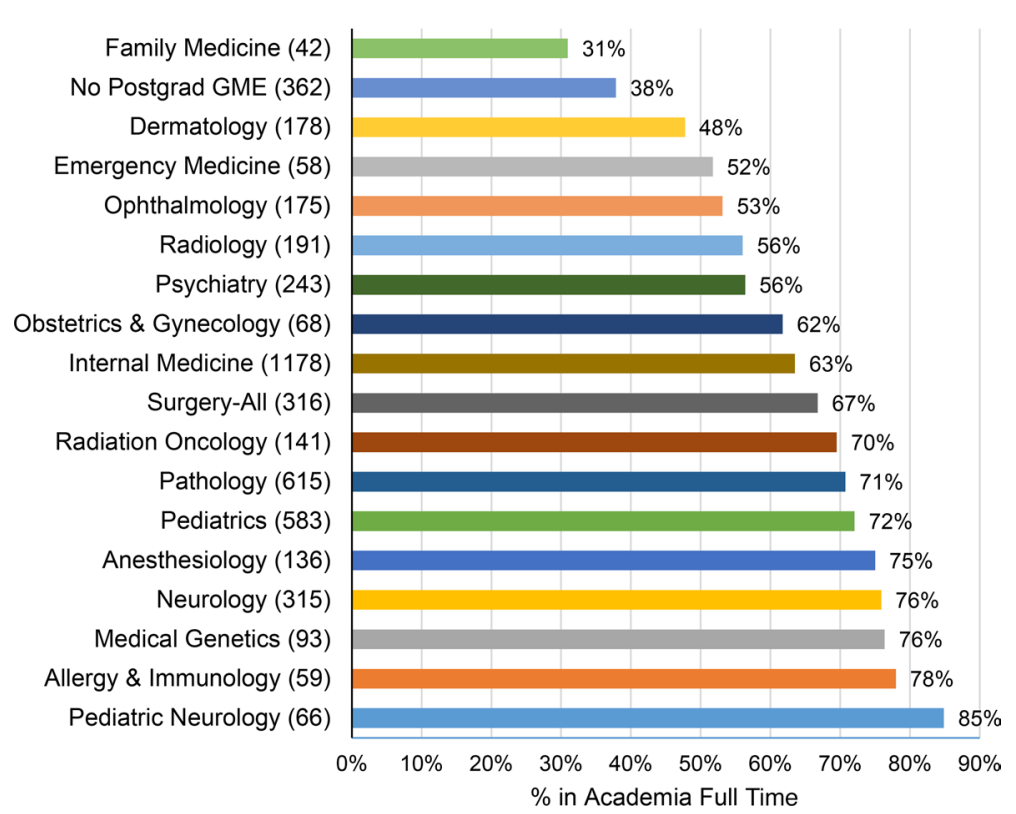

Figure 4. Current workplace as a function of graduate medical education (GME) specialty choice. Percent of alumni with a current workplace in academia full-time based on their GME specialty. Numbers in parenthesis indicate the number of individuals training in the indicated specialty, and the percentages to the right of each bar indicates the percentage with current positions in academia full-time. The total number of unique individuals is 4,819 .

and surgery taking the longest (Figure 5C). Supplemental Figure 2B and Supplemental Figure 3B show the average time to first position in academia subdividing the internal medicine and pediatrics data by subspecialty.

Residency field choice and research effort. As an additional comparison, we determined the fraction of all survey responders (not just those in academia) who reported devoting at least half of their professional time to research and broke the data down by medical specialty. The results ranged from only $10 \%$ of those in family medicine and orthopedic surgery to over $50 \%$ of those in neurology, internal medicine, pediatrics, and pediatric neurology (Figure 6A). Supplemental Figure 2C and Supplemental Figure 3C show the breakdown based on subspecialty for those in academia who trained in internal medicine and pediatrics.

Figure 6 also breaks down individual research effort by clinical specialty. Among internal medicine, neurology, pediatrics, and psychiatry, the results are remarkably similar; however, pathology is an outlier with lower research effort reported for the majority of alumni who trained in pathology (Figure 6B). Figure 6C compares research effort between those with training in orthopedic surgery, ophthalmology, neurosurgery, and dermatology with those who trained in internal medicine. In this comparison of fields, neurosurgery and orthopedic surgery are notable for $\mathrm{MD}-\mathrm{PhD}$ alumni, reporting much less time devoted to research.

Finally, because the data show an increase in overall training duration, we asked whether there is a relationship between either time to degree or time to first position in academia and subsequent research effort. Figure 7 shows the results for the 1995-2004 graduation cohort who are in academia full-time. The data show that, for survey responders, there was no substantial relationship between average research effort and time to degree, but there was a small upward trend in research effort, with increasing time to first position.

\section{Discussion}

The national MD-PhD program outcomes study provided a wealth of data on nearly everyone who has graduated from an $\mathrm{MD}-\mathrm{PhD}$ program in the United States. Here, we have returned to those data, asking a set of questions that space and time did not allow us to address in the original report. We were particularly interested in examining the relationship of medical specialty choices on the subsequent training and career path of program graduates. Recognizing that some of the data in the report may be used to establish reference standards for evaluating individual programs, we were also interested in determining whether the alumni who answered the survey were representative of those who did not. Finally, because the current workplace of program alumni is commonly used as a surrogate marker for training program success, we were interested in determining how well that marker reflects research engagement. 
A

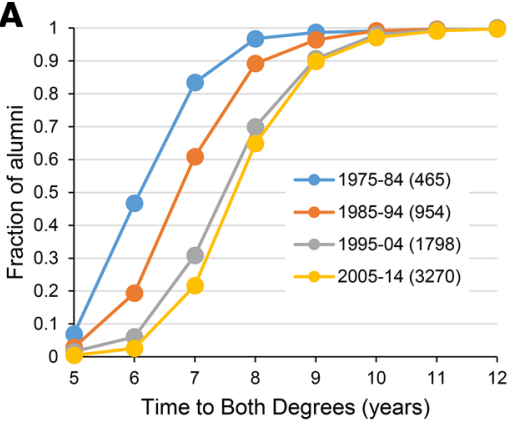

B

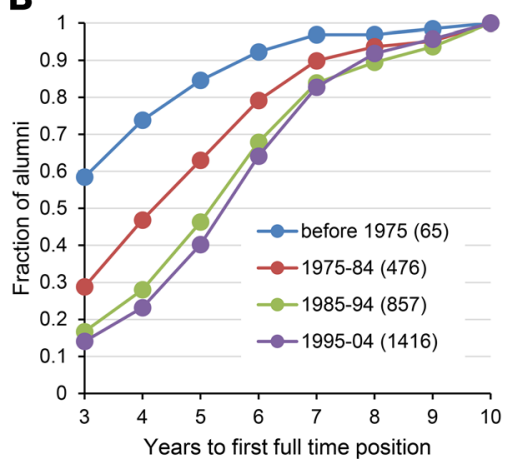

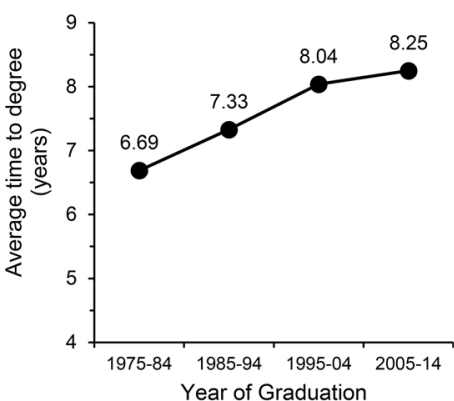

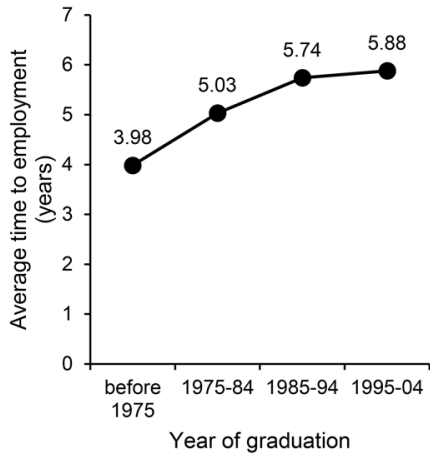

C

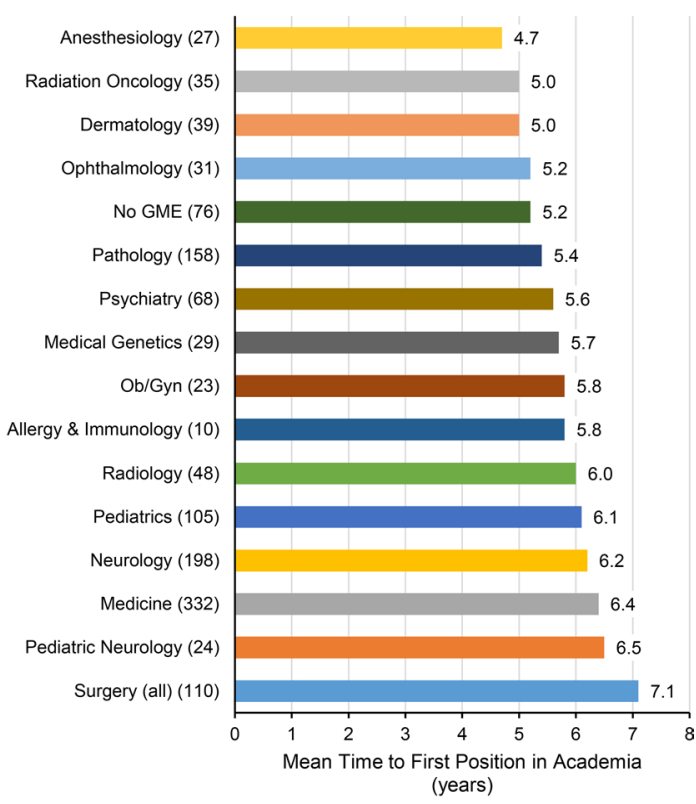

Figure 5. Time to degree and time to first full-time job. (A) (Left) Fraction of graduates who completed both degrees in the number of years indicated on the $x$ axis as a function of decade of graduation for all survey responders. The number of individuals in each cohort is listed in parentheses in the chart legend. (Right) The average time to degree in each of the graduation cohorts shown. By 1-way ANOVA using Tukey's multiple comparisons test, all data points are significantly different from each other $(P<0.001)$. (B) (Left) Time to first full-time job after completion of postgraduate training for survey responders whose first position was in either academia full-time, the NIH (or other federal agency), the pharmaceutical or biotech industries, or nongovernmental research institutes expressed as the fraction of alumni at each time. The number of individuals in each cohort is listed in parentheses in the chart legend. (B) (Right) The average time to employment in each of the graduation cohorts shown. Data for the cohort graduating between 2005-2014 were not included in this analysis because most of them were still in postgraduate training. By 1-way ANOVA using Tukey's multiple comparisons test, all of the data points are significantly different from each other; however, 1985-1994 is not significantly different from 1995-2004. (C) Mean time to first job for the 1,294 individuals who graduated between 1995-2004 with a first position in academia full-time as a function of GME specialty. The specialties are rank ordered by average time to first job.

Relationships between residency choices, careers in academia, and research effort. Over $90 \%$ of MD-PhD program graduates have chosen to do residency training, regardless of the amount of time that they eventually devote to clinical care. Approximately $60 \%$ of graduates have chosen internal medicine, pediatrics, pathology, or neurology, but nearly every clinical specialty has attracted $\mathrm{MD}-\mathrm{PhD}$ program alumni. However, the distribution of $\mathrm{MD}-\mathrm{PhD}$ program graduates among specialties is not the same as that of MD-only graduates. A study by Andriole et al. of 1,833 MD-PhD program trainees that graduated from 2000-2006 found that $\mathrm{MD}-\mathrm{PhD}$ program graduates were more likely to choose pathology and less likely to choose surgery, emergency medicine, or family medicine - a conclusion supported by the present study as well (9). A comparison with National Residency Match Program (NMRP) data (18) shows that MD-PhD program alumni currently in postgraduate training are more likely to choose pediatric neurology, medical genetics, pathology, radiation oncology, neurology, dermatology, ophthalmology, allergy and immunology, internal medicine, or radiology and less likely to choose psychiatry, anesthesiology, surgery-all, physical medicine and rehabilitation, obstetrics and gynecology, pediatrics, emergency medicine, or family medicine than medical school graduates in general. Note, however, that the $\mathrm{MD}-\mathrm{PhD}$ alumni in the national $\mathrm{MD}-\mathrm{PhD}$ program outcomes study graduated between 2005 and 2014, while the NMRP data are from 2018-2019.

The data gathered for the present study also show that a much higher proportion of $\mathrm{MD}-\mathrm{PhD}$ program alumni have moved on to faculty positions than medical school graduates in general (19, 20). Among those in academia, we found considerable variation in how MD-PhD program alumni report spending their time, with some devoting most of their time to research, while others are largely involved in patient care. An analysis suggests that the challenges of and expectations for a research career vary considerably among clinical fields, with the proportion of individuals who devote at least 

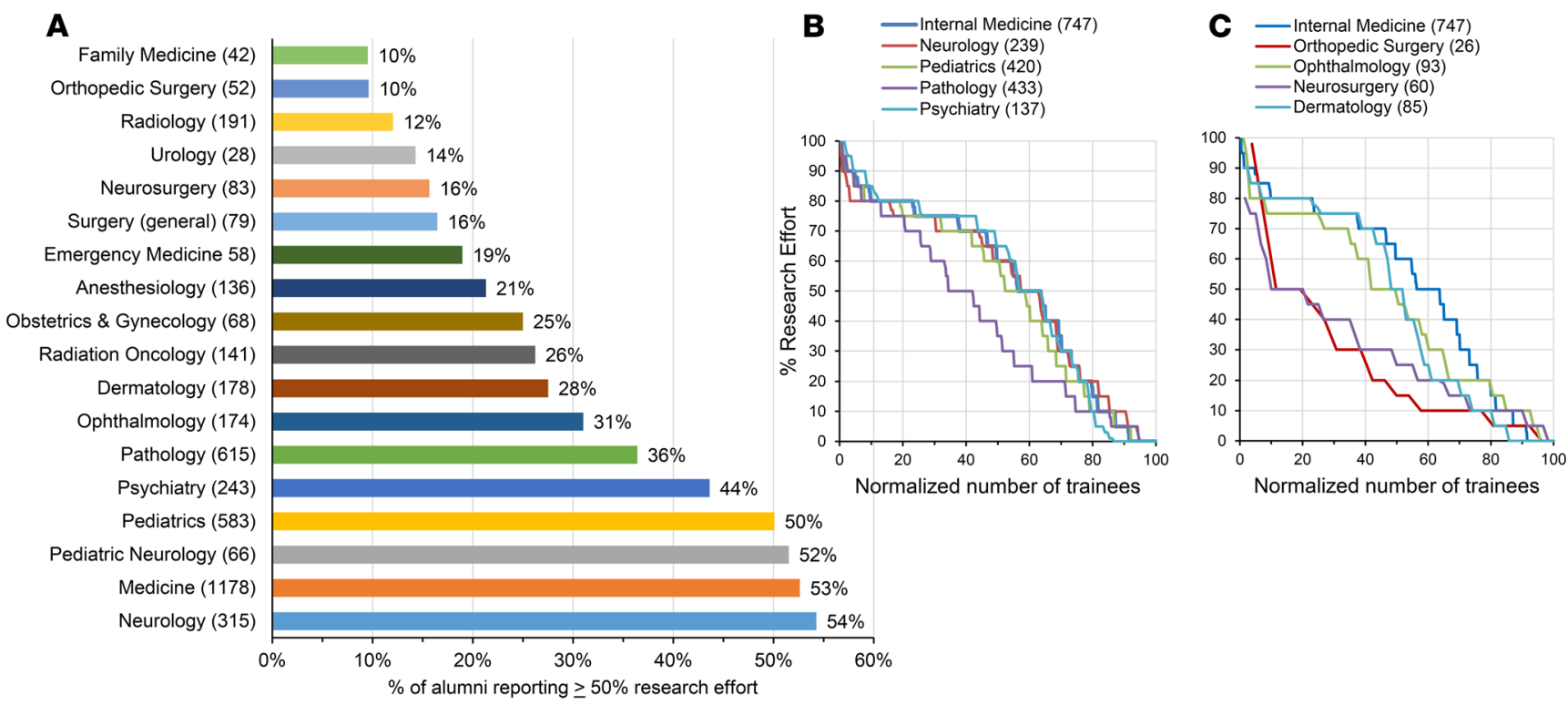

Figure 6. Medical specialty choice and research effort. (A) Percent of all survey responders out of training who reported $\geq 50 \%$ research effort as a function of GME specialty. The number of alumni training in the specialty are indicated in parentheses next to the specialty, and the percentage reporting $\geq$ $50 \%$ research effort is indicated to the right of each bar. (B and $\mathbf{C}$ ) Reported research effort as a function of GME specialty choice. Alumni are rank ordered from greatest reported percent research effort to least. The $x$ axis has been normalized by the number of alumni to allow comparisons between specialties that vary considerably in the number of alumni in each ( $n$ values are in parentheses). $P$ values derived by 1-way ANOVA with Dunnett's multiple comparisons test indicate that orthopedic surgery $(P<0.0001)$, ophthalmology $(P=0.0301)$, neurosurgery $(P<0.0001)$, and pathology $(P<0.0001)$ are significantIy different from internal medicine, while neurology $(P=0.7778)$, pediatrics $(P=0.1550)$, psychiatry $(P=0.9882)$, and dermatology $(P=0.0533)$ are not.

half of their effort to research ranging from $10 \%-54 \%$. However, even in disciplines that have attracted the largest proportion of $\mathrm{MD}-\mathrm{PhD}$ alumni, there is an entire spectrum in research effort, with some alumni devoting all or nearly all of their time to research, while others have not. We found no correlation among clinical disciplines between the proportion of graduates who eventually chose a career in academia and the proportion of graduates who devote at least half of their time to research. For example, a similarly high proportion of alumni who trained in medical genetics $(76 \%)$ and anesthesiology (75\%) reported working in academia full-time, but $67 \%$ of the medical geneticists and only $21 \%$ of the anesthesiologists reported $\geq 50 \%$ research effort. The critical question that cannot be answered from the survey data is the extent to which the choice of a specialty causes a turn from research rather than reflecting a decision to turn from research for reasons other than the choice of specialty. We suspect that both things happen.

Total training duration continues to increase and varies among clinical fields. Training duration is the sum of both the time to degree and the years of postgraduate training spent as a resident, fellow, and/or postdoc before obtaining an independent position. The national $\mathrm{MD}-\mathrm{PhD}$ program outcomes study data show that training duration at both stages continues to grow. This trend, along with an increase in the average age at matriculation, helps to account for the increase in age at the time of first faculty appointment noted in the Physician-Scientist Workforce Report (21). Notably, we found no evidence that spending more time as an $\mathrm{MD}-\mathrm{PhD}$ student resulted in greater research effort years later, when alumni became faculty.

We found considerable variation in time to first job in academia between clinical disciplines. This raises a question of whether greater integration of clinical training and research during residency/fellowship would reduce the time away from research and accelerate the transition to independence (22). We did observe an upward trend in research effort as a function of the time from graduation to a first position in academia for those who graduated between 1995-2004. This observation deserves further study, as does the question of whether the growth in training duration is, in fact, undesirable since it may deter undergraduates from applying without conferring benefits to those who do enter this career (23). With that in mind, we note with concern that the number of $\mathrm{MD}-\mathrm{PhD}$ program applicants has been essentially flat during a time when medical school applications have increased (24-26). 
A

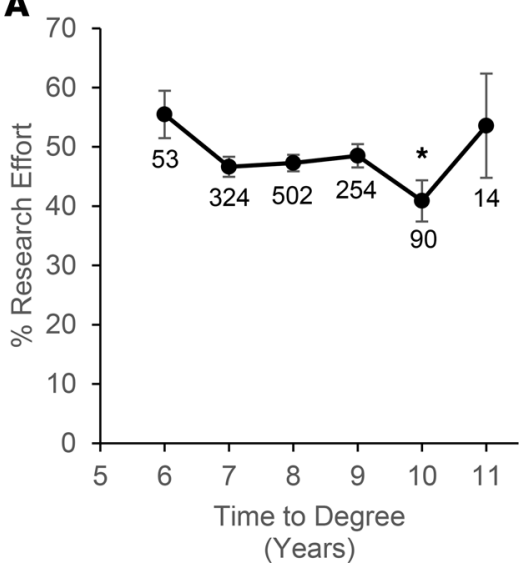

B

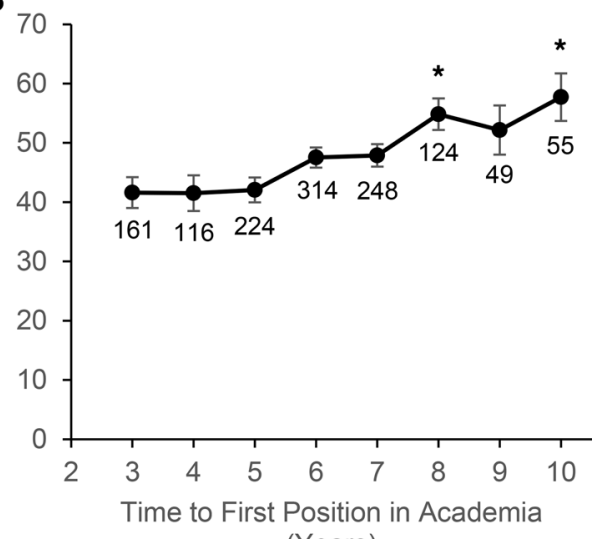

(Years)

Figure 7. Relationships between research effort and time to degree and time to first position in academia. (A) Comparison of research effort and time to degree for alumni in the 1995-2004 graduation cohort. (B) Comparison of research effort and time to first position in academia for alumni in the 1995-2004 graduation cohort. ${ }^{*} P<0.05$ compared with a 6-year time to degree (A) or a 3-year time to first position (B) by 1-way ANOVA using Dunnett's multiple comparisons test. The numbers below each data point indicate the number of alumni in the group.

Current workplace as an outcomes metric. Current workplace has become a widely used metric for evaluating $\mathrm{MD}-\mathrm{PhD}$ programs. By this metric, successful programs are those with a high proportion of graduates in academia, research institutes, and industry and a low proportion of graduates in full-time private practice, but what is the average distribution of all graduates among these different work places? The national MD-PhD program outcomes study only provided first and current position categorization for survey responders. To establish a comprehensive picture of the current positions of all alumni, we obtained aggregated, deidentified data from program directors on the current positions of half of the nonresponders. Those data show that a higher proportion of nonresponders are in private practice and a lower proportion are in academia. Combining data from the nonresponders and responders produced the numbers in Figure 1C. We suggest that these are the numbers that should be used as comparators when program outcomes are being assessed.

A separate question is whether "current position in academia" is a suitable metric for judging program success. Using the survey data, we show that there is a broad distribution of research effort among alumni in academia. We suggest that a more meaningful metric should include not only workplace, but also research effort and success in obtaining research funding. However, it is important to note that NIH funding alone is not a suitable metric for funding success. The survey data included in the AAMC report show that substantial numbers of $\mathrm{MD}-\mathrm{PhD}$ program alumni have research support from sources other than the NIH (14).

In summary, this additional analysis of the data from the national $\mathrm{MD}-\mathrm{PhD}$ program outcomes study supports 3 major conclusions. The first is that medical specialty choices affect critical aspects of the physician-scientist career path, including where people work, the time away from research, the time to first independent position, and the amount of time available to focus on research. The second is that total training duration and not just time to degree is increasing steadily. Combined with the recent trend we have seen in $\mathrm{MD}-\mathrm{PhD}$ program applicants doing gap years before starting medical school, this means that the average age of first position in academia is likely to continue to rise unless a concerted effort is made to reconsider the factors that have caused total training duration to increase. The final conclusion is that "current workplace" and NIH research awards are, by themselves, inadequate as surrogate markers for program success, since they mask important differences in how alumni divide their time between research and other activities, where they obtain research support, and how much impact they have had in the twin realms of biomedical research and patient care.

\section{Acknowledgments}

We thank Judy Shea, Professor of Medicine, University of Pennsylvania, for helpful discussions about the statistical analysis methods used in this manuscript. We also gratefully acknowledge the contributions of Irena Tartakovsky, Herschel Alexander, and Ross McKinney, of the AAMC, in the conduct of the alumni survey that was included in the National MD-PhD Outcomes Study, which provided data for this manuscript, and for their helpful feedback on an earlier version of the manuscript. We thank Maggie Krall, University of 
Pennsylvania, for helpful feedback on an earlier version of this manuscript. The conclusions and opinions expressed here are those of the authors and do not reflect policies or opinions of the AAMC. Finally, we thank all $\mathrm{MD}-\mathrm{PhD}$ program directors and administrators who supported this study by providing data and encouraging their alumni to participate in the survey, along with all of the alumni who chose to do so.

Address correspondence to: Lawrence F. Brass, University of Pennsylvania Perelman School of Medicine, Room 815 BRB-II/II, 421 Curie Boulevard, Philadelphia, Pennsylvania 19104, USA. Phone: 215.573.3540; Email: brass@Pennmedicine.upenn.edu. Or to: Myles Akabas, Albert Einstein College of Medicine, 1300 Morris Park Avenue, Bronx, New York 10461, USA. Phone: 718.430.3360; Email: myles.akabas@einstein.yu.edu.

1. Rosenberg LE. MD/PhD programs--a call for an accounting. JAMA. 2008;300(10):1208-1209.

2. Ley TJ, Rosenberg LE. The physician-scientist career pipeline in 2005: build it, and they will come. JAMA. 2005;294(11):1343-1351.

3. Wyngaarden JB. The clinical investigator as an endangered species. NEngl J Med. 1979;301(23):1254-1259.

4. Schafer AI. The vanishing physician-scientist? Transl Res. 2010;155(1):1-2.

5. Milewicz DM, Lorenz RG, Dermody TS, Brass LF, National Association of MD-PhD Programs Executive Committee. Rescuing the physician-scientist workforce: the time for action is now. J Clin Invest. 2015;125(10):3742-3747.

6. Harding CV, Akabas MH, Andersen OS. History and Outcomes of 50 Years of Physician-Scientist Training in Medical Scientist Training Programs. Acad Med. 2017;92(10):1390-1398.

7. Brass LF, Akabas MH, Burnley LD, Engman DM, Wiley CA, Andersen OS. Are MD-PhD programs meeting their goals? An analysis of career choices made by graduates of $24 \mathrm{MD}-\mathrm{PhD}$ programs. Acad Med. 2010;85(4):692-701.

8. Andriole DA, Jeffe DB. Predictors of full-time faculty appointment among MD-PhD program graduates: a national cohort study. Med Educ Online. 2016;21:30941.

9. Andriole DA, Whelan AJ, Jeffe DB. Characteristics and career intentions of the emerging MD/PhD workforce. JAMA. 2008;300(10):1165-1173.

10. McClellan DA, Talalay P. M.D.-Ph.D. training at the Johns Hopkins University School of Medicine, 1962-1991. Acad Med. 1992;67(1):36-41.

11. Frieden C, Fox BJ. Career choices of graduates from Washington University's Medical Scientist Training Program. Acad Med. 1991;66(3):162-164.

12. Dyrbye LN, Lindor KD, LaRusso NF, Cook DA. Research productivity of graduates from 3 physician-scientist training programs. Am J Med. 2008;121(12):1107-1113

13. Kosik RO, et al. Physician Scientist Training in the United States: A Survey of the Current Literature. Eval Health Prof. 2016;39(1):3-20.

14. Akabas MH, Tartakovsky I, Brass LF. The national MD-PhD program outcomes study. American Association of Medical Colleges Reports. https://www.aamc.org/download/489886/data/nationalmd-phdprogramoutcomesstudy.pdf. Accessed September 6, 2019.

15. Akabas $\mathrm{MH}$, Brass LF. The national $\mathrm{MD} / \mathrm{PhD}$ program outcomes study: Outcomes variation by gender, race and ethnicity. JCI Insight. 2019;4(19):e133010.

16. Newton DA, Grayson MS. Trends in career choice by US medical school graduates. JAMA. 2003;290(9):1179-1182.

17. Paik JC, Howard G, Lorenz RG. Postgraduate choices of graduates from medical scientist training programs, $2004-2008$. JAMA. 2009;302(12):1271-1273.

18. [No authors listed]. The Match: Charting Outcomes in the Match: US Allopathic Seniors. National Resident Matching Program. https://www.nrmp.org/wp-content/uploads/2018/06/Charting-Outcomes-in-the-Match-2018-Seniors.pdf. Accessed September 6, 2019.

19. Morrison E, Liu C. How Long Does it Take to Proceed from an MD Degree to a Medical School Faculty Appointment? AAMC Analysis in Brief. 2015;15(7):1-2.

20. Andriole DA, et al. Variables associated with full-time faculty appointment among contemporary U.S. Medical school graduates: implications for academic medicine workforce diversity. Acad Med. 2010;85(7):1250-1257.

21. Ginsburg D, et al. NIH Physician-scientist Workforce (PSW) Working Group report 2014. National Institute of Health. https:// report.nih.gov/workforce/psw/index.aspx. Accessed September 6, 2019.

22. Dzirasa K, Krishnan RR, Williams RS. Incubating the research independence of a medical scientist training program graduate: a case study. Acad Med. 2015;90(2):176-179.

23. Kersbergen CJ, Bowen CJ, Dykema AG, Koretzky MO, Tang O, Beach MC. Student Perceptions of M.D.-Ph.D. Programs: A Qualitative Identification of Barriers Facing Prospective M.D.-Ph.D. Applicants. Teach Learn Med. 2019:1-10.

24. [No authors listed]. Applicants, First-Time Applicants, Acceptees, and Matriculants to U.S. Medical Schools by Sex, 19992000 through 2008-2009. Association of American Medical Colleges. https://www.aamc.org/download/321470/data/factstablea7_1.pdf. Published October 19, 2018. Accessed Setpember 29, 2019.

25. [No authors listed]. Applicants, First-Time Applicants, Acceptees, and Matriculants to U.S. Medical Schools by Sex, 20092010 through 2018-2019. Association of American Medical Colleges. https://www.aamc.org/download/492954/data/factstablea7_2.pdf. Published November 9, 2018. Accessed September 6, 2019.

26. [No authors listed]. MD-PhD Applicants to U.S. Medical Schools by Race/Ethnicity and State of Legal Residence, 2018-2019. Association of American Medical Colleges. https://www.aamc.org/download/321542/data/factstableb7.pdf. Published November 13, 2018. Accessed September 6, 2019. 